\title{
Comparison of dissolution profiles of Ibuprofen pellets
}

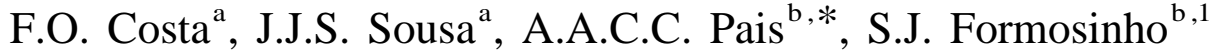 \\ ${ }^{a}$ Faculdade de Farmácia, Universidade de Coimbra, Coimbra Codex P-3004-535, Portugal \\ ${ }^{\mathrm{b}}$ Departamento de Química, Universidade de Coimbra, Coimbra Codex P-3004-535, Portugal
}

Received 13 September 2002; accepted 15 January 2003

\begin{abstract}
In this work we use both model dependent and independent techniques to assess the difference between dissolution profiles in which ibuprofen, in the form of uncoated pellets, is used as a model drug. The choice of a proper regression function, the relevance of the estimated parameters and the influence of the choice of dissolution points in the assessment of differences is discussed. The results obtained via mean dissolution times (MDT) and fit-factors $\left(f_{1}\right.$ and $\left.f_{2}\right)$ are also discussed and a non-quantitative method based on profiles correlation with graphical representation (concentration vs. concentration and rate vs. rate) presented. The tested methods discriminate similarly between curves, although not in all cases, but those based on modeling, MDT and fit-factors have shown to be less informative than the correlation approach.
\end{abstract}

(c) 2003 Elsevier Science B.V. All rights reserved.

Keywords: Dissolution profiles comparison; Model dependent methods; Model independent methods; Mean dissolution times; Fit factors; Correlation coefficients

\section{Introduction}

In vitro dissolution has been recognized for the past four decades as an important element both in drug development and quality assessment [1-8], especially in controlled release formulations. Release and further dissolution of the drug from the solid dosage forms often constitute a determining step in the in vivo absorption process and is thus used in conjunction with in vivo/in vitro correlations to establish quality control parameters. This is especial-

\footnotetext{
*Corresponding author. Tel.: +351-239-852-080; fax: +351239-827-703.

E-mail address: pais@qui.uc.pt (A.A.C.C. Pais).

${ }^{1}$ Universidade Catolica Portuguesa, Escola de Ciencias e Tecnologia, Viseu, Portugal.
}

ly relevant in checking batch to batch consistency in clinical trials, bioavailability and routine production. In the development of a new product, dissolution testing can thus aid in drug release modeling, e.g. through selection of excipients, optimization of the manufacturing process and scale-up, and formulation of test products matching required dissolution characteristics $[9,10]$. It may also be used to determine the long term stability of a dosage form and assess the impact of post-approval changes in the manufacturing process.

The methods for the comparison of dissolution profiles may be classified into two main groups according to whether or not they resort to a regression model. The model independent approaches encompass the calculation of mean dissolution times (MDT) or related characteristic values $[2,8,11,12]$, 
ANOVA-based methods [3,13] and similarity criteria based on various indexes for pairwise comparison procedures [14-19]. It should also be pointed out that some of the methods rely on the individual characterization of each curve (MDT, modeling), while others go directly into pairwise comparison (fit-factors and other similarity measures). MDT values are strongly dependent on the upper limit of the dissolution interval, while fit-factors provide exclusively a measure of absolute value discrepancy. These topics will be discussed in detail later.

In procedures requiring an underlying functional form for the description of each dissolution profile, the usual approach for the determination of the relevant parameters involves performing either ordinary linear (OLS) or non-linear least-squares (NLLS) using a limited number of typical functions [13,20-22]. In the case of OLS, a preliminary linearization step is often performed. Inspection of residuals, weighting taking account of heterocedasticy and parsimony verification are frequently absent. The problem is thus reduced to the comparison of confidence intervals for the determined fitting parameters, which requires an accurate estimation of the corresponding uncertainty. In most cases, modeling does not contemplate estimation of the predicted asymptotic value of concentration (which is not necessarily equivalent to the total dissolved amount), and results often in very poor fits. Alternative procedures for the latter task, such as the use of calibration curves in each relevant matrix, are also seldom perceived and imply the independent determination of the dose and batch consistency. However, this determination has frequently broad error ranges that should not be, in some cases, introduced in assessing profile differences. The use of qualitative (i.e. in which each profile is autonormalised) methods, based on correlation, may circumvent this difficulty. So, in this work we have applied several profile comparison methods, including correlation as a non-quantitative approach, to the dissolution data of different uncoated pellets formulations containing ibuprofen, aiming to contribute to its evaluation and identification of eventual advantages or disadvantages.

In summary, we have employed modeling resorting to some of the most popular functions for the purpose, but using weighted least squares to account for heterocedasticy, absent in previous approaches. Also introduced was an additional parameter to describe the asymptotic value of the dissolution profile. These changes not only increase the reliability of the fitting parameters but drastically reduce the sum of squares and avoids dosage determination. We have also introduced a correlation approach, complemented with the graphical representation of profiles and their time variations for assessing the respective differences and their evolution as dissolution proceeds. Comparison with traditional methods, such as those based in fit-factors and MDT values is made, and their shortcomings pinpointed.

\section{Experimental procedure}

\subsection{Materials}

In this work, we have used Ibuprofen (Shasun, batch IBU 0001097, shelf-life 12/04), as a model drug with $\mathrm{pH}$-dependent solubility and $\mathrm{p} K_{\mathrm{a}}$ of ca. 4.4 [23], microcrystalline cellulose (Microcel PH 101, Sagran, Milan, Italy), monohydrate lactose and tricalcium phosphate, as soluble and insoluble fillers respectively, and citric acid as a $\mathrm{pH}$ adjuster. Sodium hydroxide and kallium dihydrogenophosphate, analytical grade (Merck), were used in a $\mathrm{pH} 7.2 \pm 0.05$ phosphate buffer preparation. Ibuprofen solubility is increased at this $\mathrm{pH}$, when compared to more acidic ones, which facilitates the comparison of profiles.

\subsection{Preparation of pellets}

Pellets (200 g) were prepared by the extrusion/ spheronization method. Four different formulations with $20 \%$ of ibuprofen were prepared as shown in Table 1. Demineralysed water was used as the wetting liquid. The powders were mixed for $5 \mathrm{~min}$ (Kenwood, UK) before the water was added (130 $\mathrm{ml})$. The masses were extruded in an extruder with cylinders fitted with a die of $1 \mathrm{~mm}$ diameter (Caleva Process, Ltd., UK). The extrudate was spheronized in a spheronizer (G. B. Caleva, Ltd, UK) fitted with a radial plate, $250 \mathrm{~mm}$ diameter at $1020 \mathrm{rev} . / \mathrm{min}$ for $4 \mathrm{~min}$. The obtained pellets were dried in a fluidized bed dryer (Glatt), for $15 \mathrm{~min}$ at $50{ }^{\circ} \mathrm{C}$. Pellets were 
Table 1

Pellets formulations containing 20\% (w/w) of ibuprofen

\begin{tabular}{|c|c|c|c|c|c|c|c|}
\hline \multicolumn{2}{|l|}{ I } & \multicolumn{2}{|l|}{ II } & \multicolumn{2}{|l|}{ III } & \multicolumn{2}{|l|}{ IV } \\
\hline Lactose & $30.00 \%$ & Lactose & $25.00 \%$ & Tricalcium phosphate & $30.00 \%$ & Tricalcium phosphate & $25.00 \%$ \\
\hline Microcel & $50.00 \%$ & Citric acid & $5.00 \%$ & Microcel & $50.00 \%$ & Citric acid & $5.00 \%$ \\
\hline & & Microcel & $50.00 \%$ & & & Microcel & $50.00 \%$ \\
\hline
\end{tabular}

then submitted to sieve analysis using a mechanical sieve shaker for $10 \mathrm{~min}$.

\subsection{In vitro dissolution studies}

Dissolution of the size fraction $1.0-1.4 \mathrm{~mm}$ of each formulation was conducted in a USP Method 1 (rotating basket) apparatus, at a speed of 100 rev./ min, in $6 \times 900 \mathrm{ml}$ of dissolution media (phosphate buffer at $\mathrm{pH} 7.2 \pm 0.05$ ), maintained at $37 \pm 0.5^{\circ} \mathrm{C}$, using an automated assembly which consisted of a Fujitsu Ergo Proe PC, with the UV-1601PC software, a peristaltic pump (Watson Marlow 205S), a UV spectrophotometer (Shimadzu UV-1603), a cell positioner (Shimadzu CPS-Controller), a water bath fitted with a variable-speed stirrer (Vankel UK 7000) and a heater (Vankel VK750D). The released ibuprofen absorbance was recorded automatically each 5 min for $8 \mathrm{~h}$, at a wavelength of $264 \mathrm{~nm}$. Triplicates were obtained for each experiment, with a total of 18 dissolution curves for each formulation. Profile comparison methods were applied to the mean profile obtained from these 18 curves, which were previously normalized with the pellets weighted mass. Tests conducted with pellets in which the active substance is absent have shown negligible absorption in the wavelength considered.

\section{Release modeling}

Mathematical models have been used extensively for the parametric representation of the dissolution data $[3,4,13,20-22,24,25]$, which may be followed by the statistical comparison of the estimated parameters employing a $t$-test. In this work we have employed several models to compare the various profiles and also attempted to identify the nature of drug release. Several common models were analyzed (see Table 2) in a manner that slightly differs from the most common approaches. First, when the total amount released is present in the regression function, it is used as an additional parameter. Also, non-linear weighted least squares, based on the Marquardt algorithm, was employed so as to take into consideration the heterocedastic nature of the data.

In some previous approaches, the final released amount is established from the stated dose $[3,13]$.

Table 2

Release models tested

\begin{tabular}{lll}
\hline Zero order & $c_{1} t$ & Constant release rate \\
Higuchi & $c_{1} t^{0.5}$ & Fickian diffusion mechanism \\
Korsmeyer-Peppas & $c_{2} t^{c_{1}}$ & Diffusion based mechanisms \\
Hixson-Crowell & $c_{2}\left(1-\left(1-c_{1} t\right)\right)^{3}$ & Erosion release mechanism \\
First order & $c_{2}\left(1-\exp \left(-c_{1} t\right)\right)$ & First order mass balance \\
Weibull & $c_{3}\left(1-\exp \left(-\frac{t}{c_{1}}\right)^{c_{2}}\right)$ & Life-time distribution function \\
& & \\
Logistic & $c_{3} \times \frac{\exp \left(c_{1}+c_{2} \log t\right)}{1+\exp \left(c_{1}+c_{2} \log t\right)}$ & S-shaped model \\
\hline
\end{tabular}


This is common practice when commercial drug products are employed. However, the value of the dose may be affected with a systematic error of up to 5 or $10 \%$, depending on the substance. Having in view the comparison of dissolution profiles, dose errors may thus be an important part of the discrepancies found. This type of error may be simply discarded if the total released amount is used as an additional fitting parameter, which corresponds to an extrapolation based on the prevailing mechanisms for the period considered and thus do not necessarily point to the actual asymptotic value of the dissolution profile. Its inclusion, apart from minimizing the referred dose errors, has an additional consequence, i.e. drastically improving the quality of the fit. This parameter acts also as a normalising constant, and should not therefore be considered in the $t$-test comparison of profiles.

Apart from comparing profiles, modeling may also provide some insight into the drug release mechanisms. We note, however, that in some cases, a single functional form is not capable of describing the whole profile. Excipients, e.g. which promote controlled release have an effect that tends to vary during the dissolution process. In these cases we obtain information that essentially describes the dominant mechanism, which is often not the same for the profiles for which a comparison is intended.

\subsection{Results and discussion}

We have fitted the various functions for dissolution times up to 60,120 and $480 \mathrm{~min}$. In the first case, only the ascending part of the curve is considered, for $120 \mathrm{~min}$ the curves are approaching the plateau and $480 \mathrm{~min}$ correspond to the whole set of points determined. In the latter case, a significant number of points in this plateau are present in the curve (Figs. 1 and 2).

We first look at our results for $60 \mathrm{~min}$, presented in Table 3. These indicate, based on the Akaike Information Criterion (AIC) [26], that the best overall function is undoubtedly the logistic (Fig. 3), although the Weibull function also presents good fits. For formulation II, unlike the others, the best fit is obtained with the first order equation. The remaining functions are not adequate for the dissolution curves corresponding to our formulations (see, e.g. Fig. 4). Thus, the best regression functions rely more on the description of shape, having not a direct connection

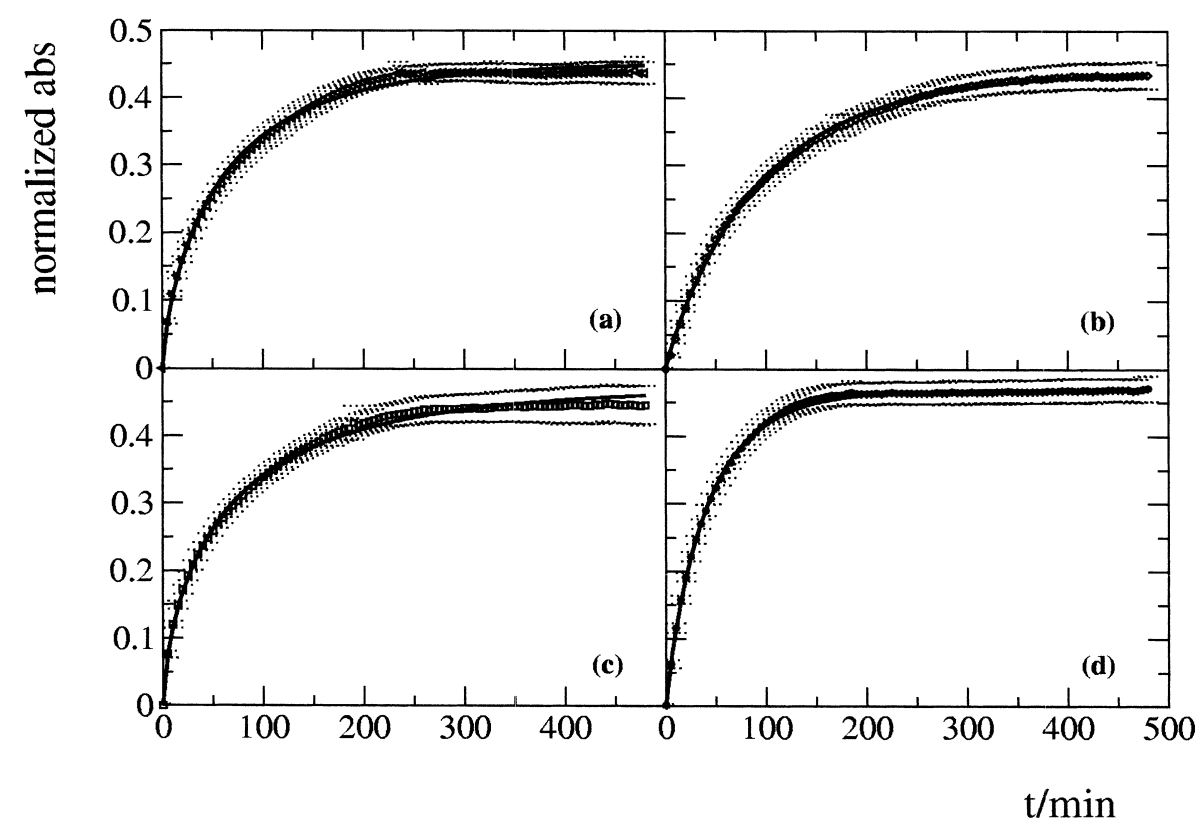

Fig. 1. Dissolution profiles of formulations I (a), II (b), III (c) and IV (d) fitted with the Weibull function (solid line). 


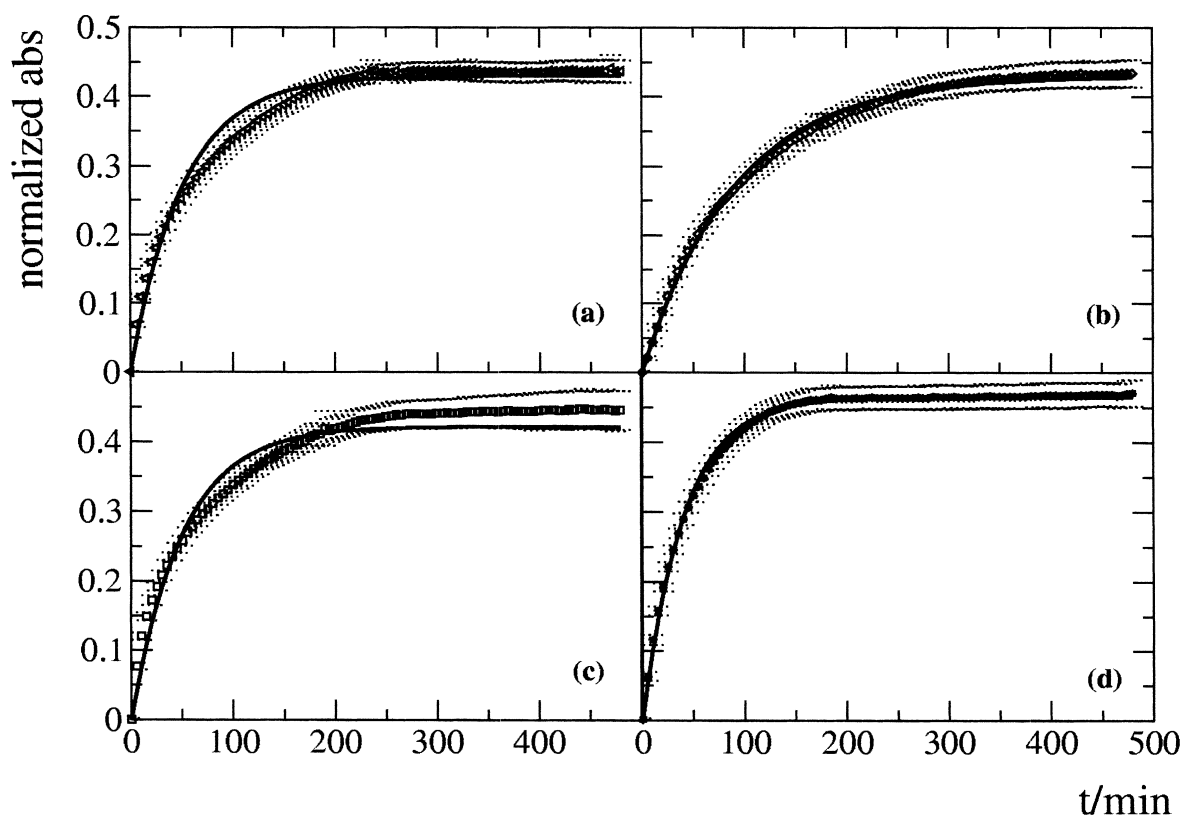

Fig. 2. As in Fig. 1 for the first order function.

with mechanism. Results for $120 \mathrm{~min}$ are similar to those reported before and stress the fact that the logistic function is more reliable for the description of the ensemble of curves (see Table 4). These findings are in agreement with some previous determinations, irrespective of the formulations under study [13].

The full curve was employed to check the prediction capabilities of functions in what concerns the asymptotic values (see Table 5). This is relevant for those functions, such as the first order one, in which this limit is built-in. Our conclusion is that the asymptotic value cannot be accurately estimated from the points corresponding to the $60 \mathrm{~min}$ period and that a substantial deviation is found even for 120 min. This may be ascribed to the way in which the mechanism evolves along the dissolution process as stated before. Also, the fact that this functions do not directly incorporate mass balance results means that they are not capable of accurately describing the plateau value which is reached after total consumption of the active substance, no matter which mechanism (or set of mechanisms) has been followed during dissolution.

Considering thus the parameters estimated from the logistic function (see Table 6 for a summary of the $t$-test results) we conclude that formulations I and III are similar, while formulation II and IV differ from reference I. Formulation IV differs significantly in both parameters for $120 \mathrm{~min}$, although for $60 \mathrm{~min}$ this difference is apparent in only one of the parameters. The other regression functions were not considered, once they fail to give a good description of the original data.

When the full curve is considered, the best fit acordding to AIC is obtained with the Weibull function (Fig. 1). The correspondent $t$-test results present, however, differences in one of the function parameters for formulations I and III. This stems from deviations between the fitted and the actual asymptotic dissolution values along the extended plateau.

\section{Model independent approaches}

Model independent approaches promote direct comparison of the dissolution data, and do not rely in the choice of model functions that sometimes may prove artificial. The objective is essentially to trans- 
Table 3

Model parameters and AIC values for $t=60 \mathrm{~min}$

\begin{tabular}{|c|c|c|c|c|c|}
\hline Function & Formulation & $c_{1}$ & $c_{2}$ & $c_{3}$ & AIC \\
\hline \multirow[t]{4}{*}{ Zero order } & I & $5.45 \times 10^{-3} \pm 5.74 \times 10^{-5}$ & - & - & 24.81 \\
\hline & II & $3.52 \times 10^{-3} \pm 5.68 \times 10^{-5}$ & - & - & 7.86 \\
\hline & III & $4.94 \times 10^{-3} \pm 5.51 \times 10^{-4}$ & - & - & 22.75 \\
\hline & IV & $7.00 \times 10^{-3} \pm 6.50 \times 10^{-5}$ & - & - & 23.42 \\
\hline \multirow[t]{4}{*}{ Higuchi } & I & $3.24 \times 10^{-2} \pm 3.34 \times 10^{-4}$ & - & - & 8.94 \\
\hline & II & $1.99 \times 10^{-2} \pm 3.35 \times 10^{-4}$ & - & - & 23.27 \\
\hline & III & $3.14 \times 10^{-2} \pm 3.45 \times 10^{-4}$ & - & - & 9.24 \\
\hline & IV & $4.28 \times 10^{-2} \pm 3.93 \times 10^{-4}$ & - & - & 14.35 \\
\hline \multirow[t]{4}{*}{ Korsmeyer-Peppas } & I & $0.59 \pm 1.83 \times 10^{-2}$ & $2.4 \times 10^{-2} \pm 1.54 \times 10^{-3}$ & - & 3.1 \\
\hline & II & $1.02 \pm 3.85 \times 10^{-2}$ & $3.26 \times 10^{-3} \pm 4.64 \times 10^{-4}$ & - & 10.35 \\
\hline & III & $0.59 \pm 2.13 \times 10^{-2}$ & $2.27-10^{-2} \pm 1.76 \times 10^{-3}$ & - & 6.21 \\
\hline & IV & $0.63 \pm 1.91 \times 10^{-2}$ & $6.9 \times 10^{-3} \pm 1.82 \times 10^{-3}$ & - & 8.2 \\
\hline \multirow[t]{4}{*}{ First order } & I & $3.87 \times 10^{-2} \pm 2.42 \times 10^{-3}$ & $0.27 \pm 8.81 \times 10^{-3}$ & - & 2.5 \\
\hline & II & $1.99 \times 10^{-3} \pm 2.44 \times 10^{-3}$ & $1.84 \pm 2.13 \times 10^{-3}$ & - & -5.5 \\
\hline & III & $3.65 \times 10^{-2} \pm 2.59 \times 10^{-3}$ & $0.27 \pm 9.29 \times 10^{-3}$ & - & -3.63 \\
\hline & IV & $3.04 \times 10^{-2} \pm 2.05 \times 10^{-3}$ & $0.40 \pm 1.52 \times 10^{-3}$ & - & 13.36 \\
\hline \multirow[t]{4}{*}{ Hixson-Crowell } & I & $1.32 \times 10^{-2} \pm 6.18 \times 10^{-4}$ & $0.24 \pm 5.90 \times 10^{-3}$ & - & 6.37 \\
\hline & II & $1.02 \times 10^{-3} \pm 1.14 \times 10^{-3}$ & $1.21 \pm 1.29 \times 10^{-3}$ & - & 10.25 \\
\hline & III & $1.25 \times 10^{-2} \pm 6.56 \times 10^{-4}$ & $0.24 \pm 5.94 \times 10^{-3}$ & - & 2.61 \\
\hline & IV & $1.10 \times 10^{-2} \pm 5.60 \times 10^{-4}$ & $0.35 \pm 9.76 \times 10^{-3}$ & - & -3.8 \\
\hline \multirow[t]{4}{*}{ Weibull } & I & $43.9 \pm 15.65$ & $0.81 \pm 7.09 \times 10^{-2}$ & $0.35 \pm 5.98 \times 10^{-2}$ & -10.72 \\
\hline & II & $35.2 \pm 3.69$ & $1.59 \pm 0.13$ & $0.21 \pm 1.75 \times 10^{-2}$ & -1.31 \\
\hline & III & $33.2 \pm 7.677$ & $0.91 \pm 8.11 \times 10^{-2}$ & $0.30 \pm 3.32 \times 10^{-2}$ & -4.32 \\
\hline & IV & $34.9 \pm 6.21$ & $0.97 \pm 7.12 \times 10^{-2}$ & $0.42 \pm 3.93 \times 10^{-2}$ & -12.10 \\
\hline \multirow[t]{4}{*}{ Logistic } & I & $-3.48 \pm 0.11$ & $0.87 \pm 9.58 \times 10^{-2}$ & $0.49 \pm 0.11$ & -12.29 \\
\hline & II & $-6.33 \pm 0.42$ & $1.78 \pm 0.19$ & $0.27 \pm 3.32 \times 10^{-2}$ & -5.09 \\
\hline & III & $-3.58 \pm 0.14$ & $1.01 \pm 0.12$ & $0.39 \pm 6.10 \times 10^{-2}$ & -6.26 \\
\hline & IV & $-3.88 \pm 0.13$ & $1.06 \pm 0.10$ & $0.55 \pm 7.55 \times 10^{-2}$ & -18.50 \\
\hline
\end{tabular}

late either the profile or profile differences into a single value.

\subsection{Moments of the dissolution profiles}

The mean in vitro dissolution times (MDT) are given from a release $M(t)$ by [2]

$\mathrm{MDT}=\frac{\int_{0}^{\infty} t \mathrm{~d} M(t)}{\int_{0}^{\infty} \mathrm{d} M(t)}$ where the denominator corresponds to the total amount dissolved, $M(\infty)$. A fraction of drug release, $M(t) / M(\infty)$ is related to the number of molecules of drug substance released from the dosage form up to the time $t$ and can be regarded as a cumulative function $F(t)$ in the statistical sense [12]. In practice, the integrals in the above equation are computed numerically, yielding,

$\mathrm{MDT}=\frac{\sum_{i} \bar{t}_{i} \Delta M_{i}}{\sum_{i} \Delta M_{i}}$

where $\bar{t}_{i}$ is the midpoint of the time period during 


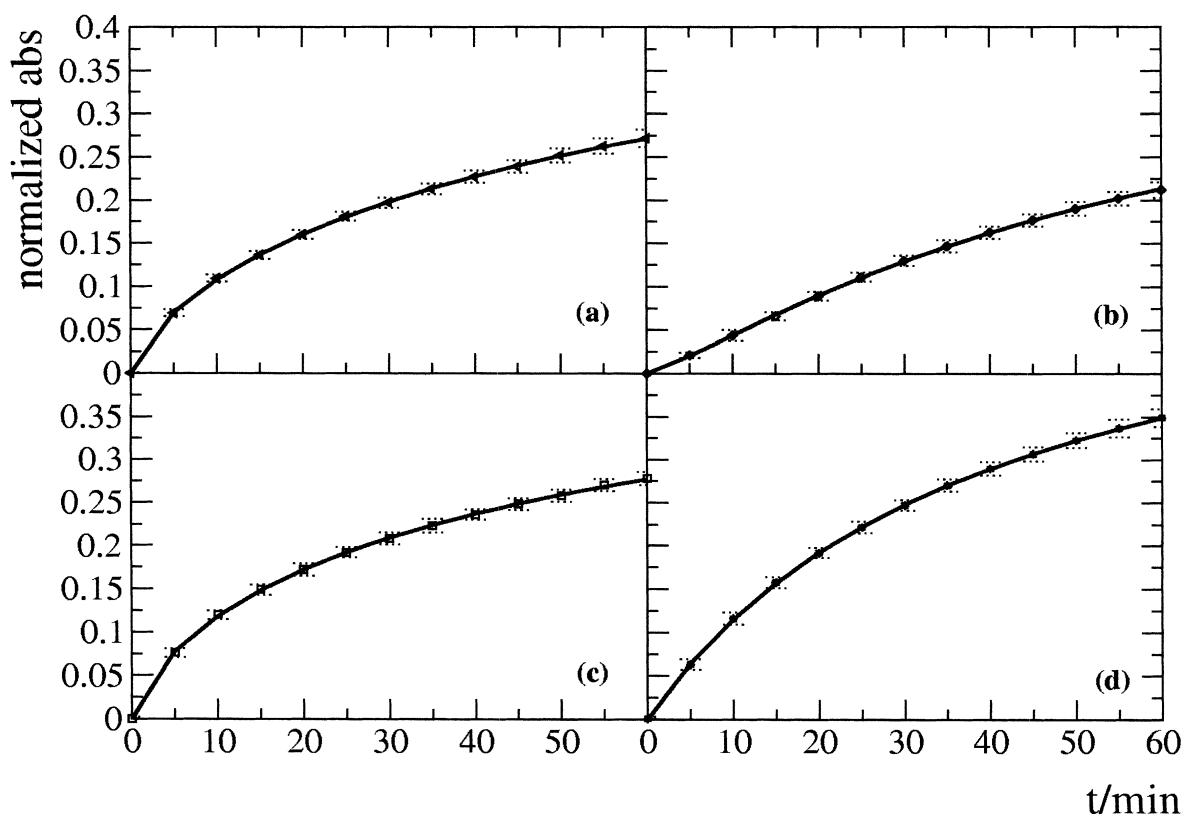

Fig. 3. Dissolution profiles up to $60 \mathrm{~min}$ fitted with the Logistic function (solid line).

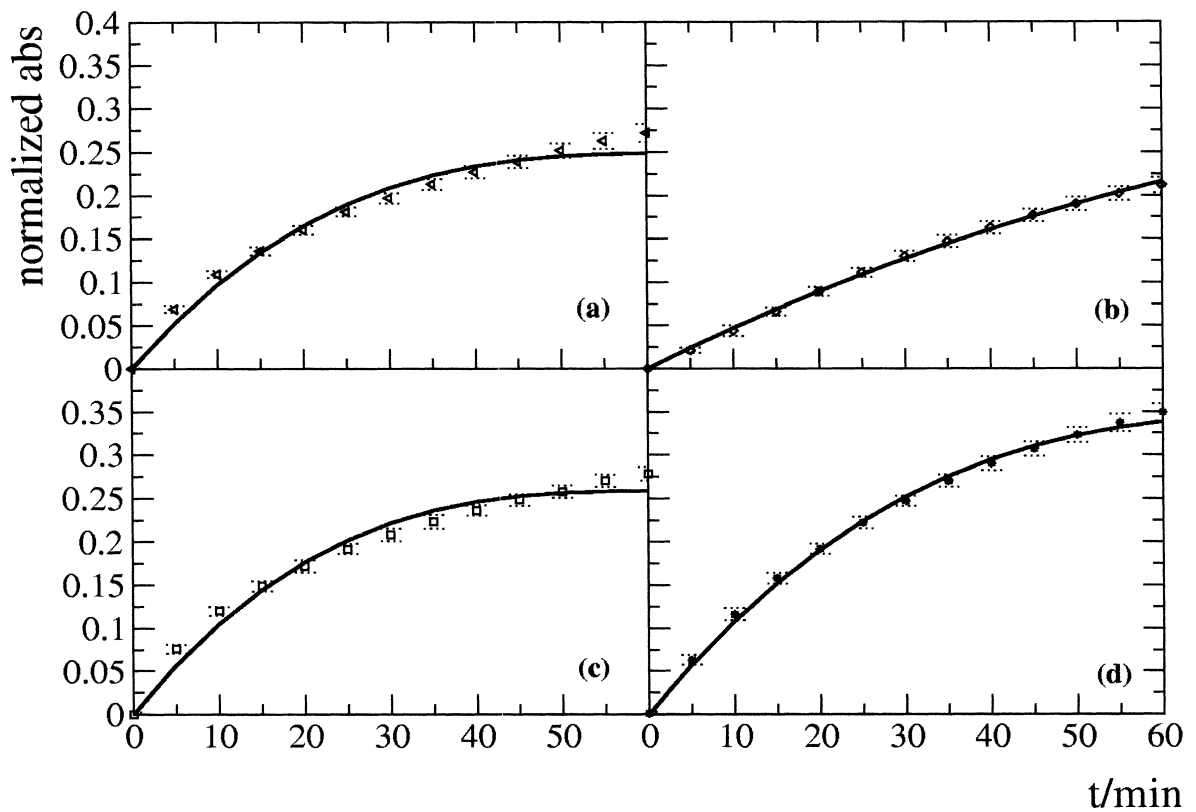

Fig. 4. As in Fig. 3 for the Hixson-Crowell function. 
Table 4

Model parameters and AIC values for $t=120 \mathrm{~min}$

\begin{tabular}{|c|c|c|c|c|c|}
\hline Function & Formulation & $c_{1}$ & $c_{2}$ & $c_{3}$ & AIC \\
\hline \multirow[t]{4}{*}{ Zero order } & I & $4.02 \times 10^{-3} \pm 3.26 \times 10^{-5}$ & - & - & 52.85 \\
\hline & II & $2.93 \times 10^{-3} \pm 2.86 \times 10^{-5}$ & - & - & 29.8 \\
\hline & III & $3.62 \times 10^{-3} \pm 2.75 \times 10^{-5}$ & - & - & 50.66 \\
\hline & IV & $4.97 \times 10^{-3} \pm 3.17 \times 10^{-5}$ & - & - & 56.31 \\
\hline \multirow[t]{4}{*}{ Higuchi } & I & $3.23 \times 10^{-2} \pm 2.51 \times 10^{-4}$ & - & - & 7.55 \\
\hline & II & $2.33 \times 10^{-2} \pm 2.31 \times 10^{-4}$ & - & - & 40.68 \\
\hline & III & $3.11 \times 10^{-2} \pm 2.29 \times 10^{-4}$ & - & - & 8.87 \\
\hline & IV & $4.22 \times 10^{-2} \pm 2.60 \times 10^{-4}$ & - & - & 19.87 \\
\hline \multirow[t]{4}{*}{ Korsmeyer-Peppas } & I & $0.53 \pm 1.09 \times 10^{-3}$ & $2.90 \times 10^{-3} \pm 1.25 \times 10^{-3}$ & - & 7.49 \\
\hline & II & $0.80 \pm 1.78 \times 10^{-3}$ & $6.89 \times 10^{-3} \pm 5.20 \times 10^{-4}$ & - & 22.32 \\
\hline & III & $0.52 \pm 1.20 \times 10^{-3}$ & $2.92 \times 10^{-3} \pm 1.46 \times 10^{-3}$ & - & 10.86 \\
\hline & IV & $0.52 \pm 9.95 \times 10^{-3}$ & $3.93 \times 10^{-3} \pm 1.61 \times 10^{-3}$ & - & 21.96 \\
\hline \multirow[t]{4}{*}{ First order } & I & $2.78 \times 10^{-3} 2.75 \times 10^{-4}$ & $0.42 \pm 1.76 \times 10^{-3}$ & - & 11.72 \\
\hline & II & $9.94 \times 10^{-3} \pm 8.43 \times 10^{-4}$ & $0.42 .50 \times 10^{-3}$ & - & 11.48 \\
\hline & III & $2.63 \times 10^{-3} \pm 1.05 \times 10^{-3}$ & $0.32 \pm 5.56 \times 10^{-3}$ & - & 7.55 \\
\hline & IV & $2.62 \times 10^{-3} 8.34 \times 10^{-4}$ & $0.46 \pm 6.29 \times 10^{-3}$ & - & -9.03 \\
\hline \multirow[t]{4}{*}{ Hixson-Crowell } & I & $8.84 \times 10^{-3} \pm 2.47 \times 10^{-4}$ & $0.31 \pm 4.34 \times 10^{-3}$ & - & 21.24 \\
\hline & II & $4.12 \times 10^{-3} \pm 2.80 \times 10^{-4}$ & $0.34 \pm 1.55 \times 10^{-3}$ & - & 10.33 \\
\hline & III & $8.24 \times 10^{-3} \pm 2.38 \times 10^{-4}$ & $0.30 \pm 3.89 \times 10^{-3}$ & - & 18.06 \\
\hline & IV & $8.42 \times 10^{-3} \pm 1.95 \times 10^{-4}$ & $0.41 \pm 4.31 \times 10^{-3}$ & - & 11.07 \\
\hline \multirow[t]{4}{*}{ Weibull } & I & $67.5 \pm 14.19$ & $0.75 \pm 3.98 \times 10^{-3}$ & $0.43 \pm 3.88 \times 10^{-3}$ & -25.10 \\
\hline & II & $55.5 \pm 3.80$ & $1.29 \pm 5.92 \times 10^{-3}$ & $0.30 \pm 1.26 \times 10^{-3}$ & -0.29 \\
\hline & III & $58.8 \pm 10.04$ & $0.78 \pm 4.40 \times 10^{-3}$ & $0.39 \pm 2.84 \times 10^{-3}$ & -11.55 \\
\hline & IV & $43.5 \pm 3.36$ & $0.91 \pm 3.83 \times 10^{-3}$ & $0.47 \pm 1.61 \times 10^{-3}$ & -24.03 \\
\hline \multirow[t]{4}{*}{ Logistic } & I & $-3.56 \pm 6.61 \times 10^{-3}$ & $0.81 \pm 5.44 \times 10^{-3}$ & $0.59 \pm 7.38 \times 10^{-3}$ & -31.29 \\
\hline & II & $-5.85 \pm 0.23$ & $1.48 \pm 8.88 \times 10^{-3}$ & $0.37 \pm 2.32 \times 10^{-3}$ & -7.09 \\
\hline & III & $-3.60 \pm 8.75 \times 10^{-3}$ & $0.87 \pm 6.24 \times 10^{-3}$ & $0.51 \pm 5.19 \times 10^{-3}$ & -16.35 \\
\hline & IV & $-3.87 \pm 0.11$ & $1.04 \pm 5.82 \times 10^{-3}$ & $0.58 \pm 3.13 \times 10^{-3}$ & -44.64 \\
\hline
\end{tabular}

which the fraction $\Delta M_{i}$ of the drug as been released from the dosage form [2]. Other similar formula gives the moments of dissolution times of order $k$

$m_{k}=\frac{\sum_{i} \bar{t}_{i}^{k} \Delta M_{i}}{\sum_{i} \Delta M_{i}}$

One of the advantages of this method is that the comparison is based on a physically significant quantity, of widespread use for establishing in vitro/ in vivo correlations. It is also possible to characterize the profiles with statistical moments such as MDT, its relative dispersion (RD) and the variance associ- ated with the MDT (VT) [12]. An additional advantage is that Eqs. (1)-(3) are self-normalized, i.e. they are amenable to direct treatment of signal data. The method may require, however, the knowledge of the time at which the plateau, i.e. full dissolution, is attained (see below).

Variance of dissolution times are estimated from

$$
\mathrm{VT}=\frac{\sum_{i}\left(\bar{t}_{i}-\mathrm{MDT}\right)^{2} \Delta M_{i}}{\sum_{i} \Delta M_{i}}
$$

The relative dispersion of dissolution times (RD) is given by [27] 
Table 5

Model parameters and AIC values for $t=480 \mathrm{~min}$

\begin{tabular}{|c|c|c|c|c|c|}
\hline Function & Formulation & $c_{1}$ & $c_{2}$ & $c_{3}$ & AIC \\
\hline \multirow[t]{4}{*}{ Zero order } & I & $1.36 \times 10^{-3} \pm 3.26 \times 10^{-5}$ & - & - & 244.03 \\
\hline & II & $1.33 \times 10^{-3} \pm 6.19 \times 10^{-6}$ & - & - & 205.83 \\
\hline & III & $1.55 \times 10^{-3} \pm 7.89 \times 10^{-6}$ & - & - & 229.26 \\
\hline & IV & $1.51 \times 10^{-3} \pm 6.13 \times 10^{-6}$ & - & - & 260.92 \\
\hline \multirow[t]{4}{*}{ Higuchi } & I & $2.50 \times 10^{-3} \pm 1.20 \times 10^{-4}$ & - & - & 157.13 \\
\hline & II & $2.29 \times 10^{-3} \pm 1.04 \times 10^{-4}$ & - & - & 112.93 \\
\hline & III & $2.61 \times 10^{-3} \pm 8.67 \times 10^{-5}$ & - & - & 126.97 \\
\hline & IV & $2.88 \times 10^{-3} \pm 1.00 \times 10^{-4}$ & - & - & 191.00 \\
\hline \multirow[t]{4}{*}{ Korsmeyer-Peppas } & I & $0.35 \pm 3.75 \times 10^{-3}$ & $5.7 \times 10^{-3} \pm 1.15 \times 10^{-3}$ & - & 114.06 \\
\hline & II & $0.50 \pm 5.42 \times 10^{-3}$ & $2.34 \times 10^{-3} \pm 6.86 \times 10^{-4}$ & - & 115.4 \\
\hline & III & $0.36 \pm 4.88 \times 10^{-3}$ & $5.34 \times 10^{-3} \pm 1.32 \times 10^{-3}$ & - & 80.91 \\
\hline & IV & $0.27 \pm 3.58 \times 10^{-3}$ & $9.96 \times 10^{-3} \pm 1.87 \times 10^{-3}$ & - & 134.23 \\
\hline \multirow[t]{4}{*}{ First order } & I & $1.78 \times 10^{-3} \pm 2.74 \times 10^{-4}$ & $0.42 \pm 1.76 \times 10^{-3}$ & - & 36.17 \\
\hline & II & $1.00 \times 10^{-3} \pm 1.92 \times 10^{-4}$ & $0.42 \pm 3.02 \times 10^{-3}$ & - & -31.44 \\
\hline & III & $1.70 \times 10^{-3} \pm 3.22 \times 10^{-4}$ & $0.40 \pm 2.27 \times 10^{-3}$ & - & 28.43 \\
\hline & IV & $2.42 \times 10^{-3} \pm 3.85 \times 10^{-4}$ & $0.46 \pm 1.98 \times 10^{-3}$ & - & -80.62 \\
\hline \multirow[t]{4}{*}{ Hixson-Crowell } & I & $3.38 \times 10^{-3} \pm 3.04 \times 10^{-5}$ & $0.42 \pm 2.09 \times 10^{-3}$ & - & 137.59 \\
\hline & II & $3.13 \times 10^{-3} \pm 4.72 \times 10^{-5}$ & $0.39 \pm 2.75 \times 10^{-3}$ & - & 8.78 \\
\hline & III & $3.55 \times 10^{-3} \pm 3.93 \times 10^{-5}$ & $0.41 \pm 2.93 \times 10^{-3}$ & - & 113.13 \\
\hline & IV & $3.44 \times 10^{-3} \pm 2.82 \times 10^{-5}$ & $0.49 \pm 2.61 \times 10^{-3}$ & - & 174.57 \\
\hline \multirow[t]{4}{*}{ Weibull } & I & $65.1 \pm 1.76$ & $0.79 \pm 1.57 \times 10^{-3}$ & $0.43 \pm 3.14 \times 10^{-3}$ & -50.44 \\
\hline & II & $98.1 \pm 2.41$ & $1.02 \pm 2.05 \times 10^{-3}$ & $0.42 \pm 3.83 \times 10^{-3}$ & -29.94 \\
\hline & III & $72.5 \pm 2.87$ & $0.76 \pm 1.90 \times 10^{-3}$ & $0.43 \pm 5.66 \times 10^{-3}$ & -89.12 \\
\hline & IV & $42.1 \pm 0.76$ & $0.93 \pm 2.07 \times 10^{-3}$ & $0.46 \pm 2.26 \times 10^{-3}$ & -142.36 \\
\hline \multirow[t]{4}{*}{ Logistic } & I & $-3.80 \pm 7.38 \times 10^{-3}$ & $0.97 \pm 2.62 \times 10^{-3}$ & $0.49 \pm 6.77 \times 10^{-3}$ & -2.60 \\
\hline & II & $-5.48 \pm 0.12$ & $1.26 \pm 3.38 \times 10^{-3}$ & $0.47 \pm 7.20 \times 10^{-3}$ & -67.27 \\
\hline & III & $-3.71 \pm 8.44 \times 10^{-3}$ & $0.91 \pm 3.09 \times 10^{-3}$ & $0.50 \pm 1.12 \times 10^{-3}$ & -60.4 \\
\hline & IV & $-4.36 \pm 0.11$ & $1.29 \pm 3.59 \times 10^{-3}$ & $0.48 \pm 3.64 \times 10^{-3}$ & -32.92 \\
\hline
\end{tabular}

$\mathrm{RD}=\frac{\mathrm{VT}}{\mathrm{MDT}^{2}}$

In spite of the mentioned advantages, the use of mean dissolution times (or similar approaches) poses some problems. For zero order (or close to zero order) release mechanisms, Eq. (1) yields, in practice, $\mathrm{MDT}=\frac{1}{2} t_{\max }$ when infinite dissolution times are replaced by specific dissolution intervals between 0 and $t_{\max }$. This means that when such curves are

Table 6

Results of the comparison of formulation I with test curves from modeling functions ( $t$-test, $\alpha=0.05)$. Indicated is when difference is found for only one parameter

\begin{tabular}{llll}
\hline Formulation & Logistic $(t=60 \mathrm{~min})$ & Logistic $(t=120 \mathrm{~min})$ & Weibull $(t=480$ min $)$ \\
\hline II & Different & Different & Different \\
III & Similar & Similar & Different (1 parameter) \\
IV & Different (1 parameter $)$ & Different & Different \\
\hline
\end{tabular}


truncated before full dissolution, the result is independent of the release rate, rendering impossible the discrimination between two curves. Of course, for other release mechanisms differences may arise, but the dependence on the upper integration limit is always present.

\subsection{Results and discussion}

Results obtained from our formulations are shown in Table 7, for $t=60,120$, and $480 \mathrm{~min}$. With the obtained RD values it is not possible to conclude about release order or, more generally, mechanism, by comparison to those obtained for some common models (see description in Ref. [12]). This fact suggests the coexistence of different mechanisms. MDT values, naturally, tend to increase with time. The increase is much more evident when the formulation has some prolonged release as we can see with formulation II when compared to the reference (formulation I). The MDT value of formulation IV at $t=60 \mathrm{~min}$ is higher than that of the reference which reflects a lower dissolution rate, but this effect is lost and the dissolution of this formulation reaches the lowest MDT value, showing a modification in release rate or dissolution mechanism. Also, we see that these values are stable even when the plateau is taken into consideration, which allows for a characterization of the complete dissolution curve [12]. These findings are also illustrated in Fig. 5. This method, for our data, indicates the extension of an induced controlled release effect even when it is observed for a very short period of time, which is very useful in formula development and comparison of excipients.

\subsection{Fit factors}

Recently a simple model independent approach

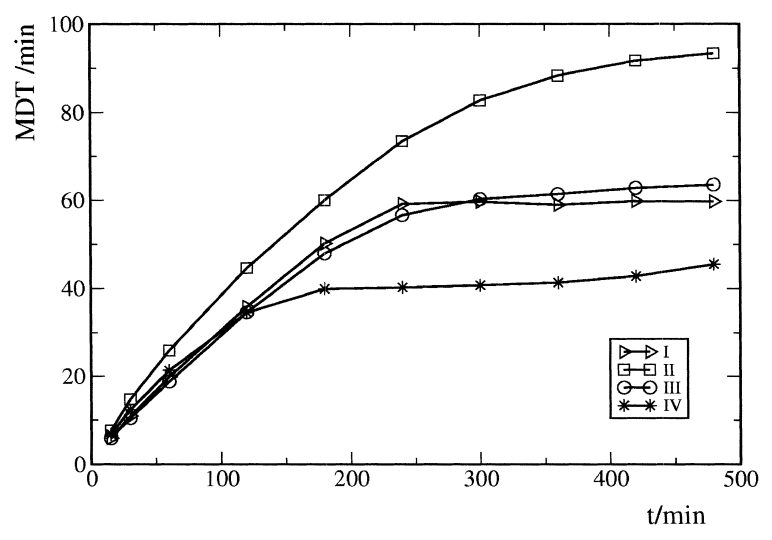

Fig. 5. MDT values for formulations I, II, III and IV at different cumulative time points.

that uses a difference factor $\left(f_{1}\right)$ and a similarity factor $\left(f_{2}\right)$ was proposed to compare dissolution profiles [14]. Fit factors were adopted by FDA Center for Drug Evaluation and Research (CDER) and the similarity factor was also adopted by the European Medicines Evaluation Agency (EMEA) Committee for Proprietary Medicinal Products (CPMP) as an assessment criterion of similarity between two in-vitro dissolution profiles $[9,10]$. The difference factor $\left(f_{1}\right)$ calculates the percent difference between the two curves at each time point and is a measure of the relative error between the two curves,

$f_{1}=\frac{\sum_{t=1}^{n}\left|R_{t}-T_{t}\right|}{\sum R_{t}} \times 100$

where $n$ is the number of time points, $R_{t}$ is the dissolution value of the reference formulation at time $t$ and $T_{t}$ is the dissolution value of the test formulation at time $t$.

The similarity factor $\left(f_{2}\right)$ is a logarithmic re-

Table 7

MDT and VT values for formulations I, II, III and IV at $\mathrm{t}=60,120$ and 480 min (number of points considered $n=13,25$ and 97 , respectively)

\begin{tabular}{|c|c|c|c|c|c|c|c|c|c|c|c|c|}
\hline & \multicolumn{4}{|l|}{$60 \mathrm{~min}$} & \multicolumn{4}{|c|}{$120 \mathrm{~min}$} & \multicolumn{4}{|c|}{$480 \mathrm{~min}$} \\
\hline & I & II & III & IV & I & II & III & IV & I & II & III & IV \\
\hline $\mathrm{MDT} / \mathrm{min}$ & 19.89 & 25.89 & 18.79 & 21.38 & 35.81 & 44.58 & 34.56 & 34.5 & 59.79 & 93.42 & 63.57 & 45.52 \\
\hline $\mathrm{VT} / \mathrm{min}^{2}$ & 1041 & 1256 & 988.9 & 768.4 & 3036 & 3469 & 3090 & 2119 & 8567 & 18684 & 11476 & 7265 \\
\hline RD & 2.631 & 1.874 & 2.801 & 1.681 & 2.368 & 1.746 & 2.587 & 1.780 & 2.396 & 2.141 & 2.840 & 3.506 \\
\hline
\end{tabular}


ciprocal square root transformation of the sum of squared error and is a measurement of the similarity in the percent $(\%)$ dissolution between the curves

$f_{2}=50 \log \left\{\left[1+\frac{1}{n} \sum_{t=1}^{n}\left(R_{t}-T_{t}\right)^{2}\right]^{-0.5} \times 100\right\}$

For curves to be considered similar, $f_{1}$ values should be close to 0 and $f_{2}$ values should be close to 100. Generally, $f_{1}$ values up to $15(0-15)$ and $f_{2}$ values greater than $50(50-100)$, which means an average difference of no more than $10 \%$ at the sample time points [16], ensures equivalence of the two curves and thus of the performance of the test and reference products [10].

Fit factors are essentially a quantitative method, reflecting the differences between corresponding values in the two curves. They do not indicate the sense of the deviation, and yield the same value irrespectively of the test curve being placed below or above the reference [14]. Thus, they do not directly take into consideration the shape of the curve, and not allow for a variation in the spacing between sampling times $[19,25]$. Also, they do not take into account variability within test and references batches. Since fit factors are commulative functions they are sensitive to measurements obtained after either test or reference batch are dissolved more than $85 \%$ [16]. Thus it becomes apparent that the selection and determination of the dissolution end pull points plays a critical role in the calculation of fit factors and the subsequent decision as to whether the test and reference profiles resemble each other or not $[13,15]$.

In spite of these limitations fit-factors have been very useful in comparing dissolution curves once they present a great advantage of reducing complexity by providing a single number to describe two curves that consist of several points [14].

Results obtained from our formulations are shown in Table 8. The recommendations presented in the above mentioned guidelines were followed and we observe that the similarity factor $f_{2}$ is more sensitive in finding dissimilarity between dissolution curves than the difference factor $f_{1}$ and, also, that these values are dependent on the length of dissolution profile and number of time points chosen. These findings have been also mentioned by other authors $[13,15]$. Our results show clear differences between formulations I and II and a similitude between I and III. In what concerns formulations I and IV the difference appears for the largest dissolution interval considered $(120 \mathrm{~min})$. This might indicate that a large number of time points should be included in order to obtain a suitable discrimination.

\subsection{Correlation coefficients and similarity criteria}

In cluster analysis [28] euclidean distances (and their variants) and correlation coefficients are frequently used as measures of similarity. These measures can be applied in a straightforward manner to dissolution curves. The use of euclidean distances would have the same underlying philosophy as the use of fit-factors, also consisting of an essentially quantitative approach. The use of correlation coefficients in the comparison focus, for our analysis, on the shape of the curves, discarding the absolute value. It is thus, a valuable complement of some other approaches.

The correlation coefficient $(\rho)$ measures the strength of the linear relationship between two variables, say $X$ and $Y$ :

Table 8

Fit factors values for formulations II, III and IV compared with formulation I

\begin{tabular}{|c|c|c|c|c|c|c|c|c|c|c|c|c|}
\hline \multirow[t]{2}{*}{ Fit factors } & \multicolumn{3}{|c|}{$t=10,40$ and $120 \mathrm{~min}$} & \multicolumn{3}{|c|}{ up to $t=30 \mathrm{~min}$} & \multicolumn{3}{|c|}{ up to $t=60 \mathrm{~min}$} & \multicolumn{3}{|c|}{ up to $t=120 \mathrm{~min}$} \\
\hline & I/II & $\mathrm{I} / \mathrm{III}$ & $\mathrm{I} / \mathrm{IV}$ & $\mathrm{I} / \mathrm{II}$ & I/III & $\mathrm{I} / \mathrm{IV}$ & $\mathrm{I} / \mathrm{II}$ & I/III & $\mathrm{I} / \mathrm{IV}$ & $\mathrm{I} / \mathrm{II}$ & $\mathrm{I} / \mathrm{III}$ & $\mathrm{I} / \mathrm{IV}^{\mathrm{a}}$ \\
\hline$f_{1}$ & 3.96 & 0.57 & 2.41 & 8.65 & 0.65 & 2.6 & 13.6 & 1.19 & 6.57 & 26.4 & 2.29 & 9.63 \\
\hline$f_{2}$ & 42.6 & 88.0 & 50.4 & 42.2 & 92.6 & 63.9 & 42.2 & 87.2 & 52.1 & 43.4 & 86.5 & 48.6 \\
\hline
\end{tabular}

For the second heading, only the three points indicated have been considered, while in the remaining, and except where noted, all determined points have been included.

${ }^{a}$ Dissolution curve of formulation IV without time points from 90 to $115 \mathrm{~min}$. 
$\rho=\frac{\sum(x-\bar{x})(y-\bar{y})}{\sqrt{\sum(x-\bar{x})^{2} \sum(y-\bar{y})^{2}}}$

where $x$ and $y$ are the variables points and $\bar{x}$ and $\bar{y}$ are the respective means. Correlation coefficient ranges from -1 to +1 . For $\rho= \pm 1$ there exists a functional relationship between $X$ and $Y$ and all points $(x, y)$ lie on a straight line. If $\rho=0$ then $X$ and $Y$ are uncorrelated; two random variables are the more strongly correlated the closer $|\rho|$ is to 1 [29].

\subsubsection{Graphical representation}

The use of correlation coefficients suggests the concomitant use of concentration vs. concentration or rate vs. rate plots. In fact, the linearity of these plots corresponds, using this criterium, to a high similitude between curves. These plots also provide information concerning the relative evolution of profiles and, for complete dissolution curves, pinpoint the times for which dissolution has come to a halt in certain formulation while continues in others.

\subsubsection{Discussion}

Our results are based on correlation coefficients calculated both from the normalized signals and the corresponding rates, respectively presented in Fig. 6 panels (a) and (b). While rate based coefficients emphasize differences, those related to concentration show the commulative behavior of the dissolution profiles, which is also shown in Fig. 7. Both co-

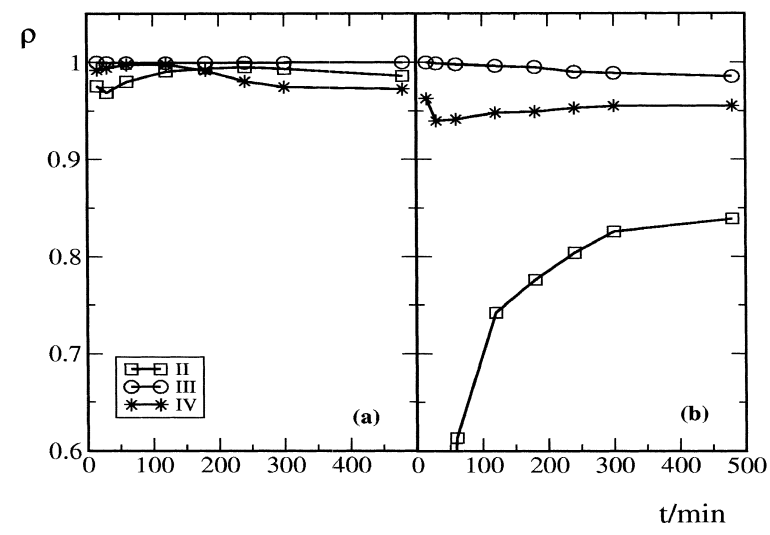

Fig. 6. Correlation coefficients between formulations pairs (reference/test) I/II, I/III and I/IV normalized absorvance signal (a) and respective time derivatives (b).

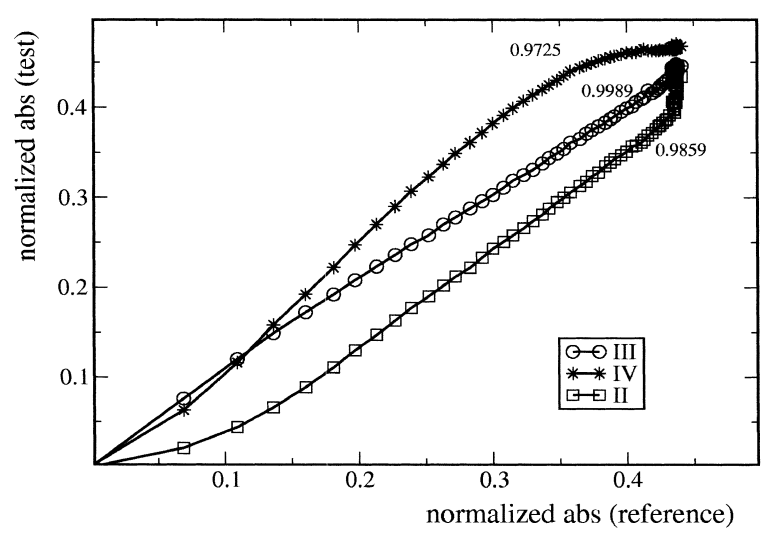

Fig. 7. Graphical representation of test formulations II, III and IV normalized absorvance signal as a function of that of formulation I (reference). Correlation coefficients $(t=480 \mathrm{~min})$ also shown.

efficients clearly present a high degree of similitude for formulation III and the reference that decreases slightly for formulation IV and again, more markedly, for formulation II. This picture is present up to ca. $120 \mathrm{~min}$, where a substantial portion the active substance is already in solution, and inverts for formulation II and IV when a large number of points in the asymptotic plateau is included. We note that, for our data, the choice of time at which the curve is truncated becomes irrelevant with rate coefficients.

In what concerns the concentration plots, Fig. 7, we see that the formulation IV is the first to attain complete dissolution, while formulation II is the slowest. It also patent the similitude between formulation I and III. We may also observe that the bulk concentration for formulation II is always below that of the other curves. For formulation IV, a slower start is compensated by an intermediate increase.

The rate vs. rate plots, Fig. 8, depict similar conclusions. The effects in the beginning of the curves are much more emphasized, due to drastic initial variations and in spite of our constant interval between time points. The initial zero order release present for formulation II is clearly represented as is the initially slow behavior of formulation IV and its subsequent increase.

\section{Final remarks}

All methods considered here have given results 


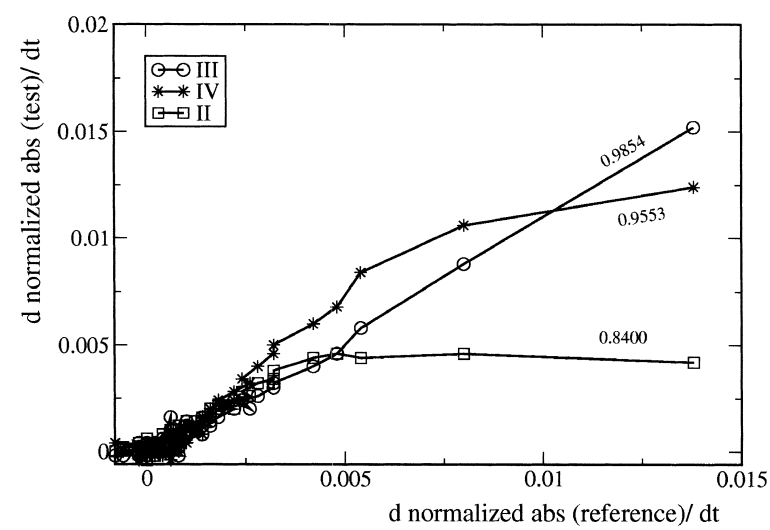

Fig. 8. Graphical representation of test formulations II, III and IV normalized signal time derivative as a function of the corresponding values for the reference. Correlation coefficients $(t=480$ min) are also shown.

with some consistency, especially when dissolution percentages are typically below $80 \%$. When points in the asymptotic plateau are considered, some differences may be found between methods. In this regards, MDT values and, especially, correlation coefficients based on signal time derivatives seem to be the more stable approaches.

The methods differ in complexity and user friendliness and the information provided on the dissolution profiles varies. Modeling is a useful characterization of mechanism, but poses some problems. Generally, good quality fits are obtained with shape rather than mechanism-based functions, mostly due to a change in mechanism as the dissolution proceeds or to a coexistence of concomitant mechanisms.

MDT values are an useful way to reduce each curve to a single number, which may be related in some cases to the dissolution rate constant. Mechanisms may also be inferred in favorable situations. However, its importance for practical use is somewhat diminished by the fact that discrimination is, formally, dependent on the final time point considered, i.e. their proper use requires a detailed knowledge of the dissolution end-point.

Fit-factors are both robust and reliable, but strongly focused in quantitative differences, mostly overlooking the similitude of shapes. Also, they do not take into consideration the variability within each curve.

Finally, the use of correlation coefficients and the corresponding graphical representation provides a useful way to emphasize non-quantitative differences, and their evolution along the profile. However, they lack a simple criterion for classification into 'similar' or 'different'.

In our view, the combination of fit-factors, in a quantitative perspective, with correlation coefficients, for spotting shape differences, provides a reliable way to assess differences between profiles.

If one is interested in an in-depth analysis of the release-control factors, the time extension of their action and its sense, then suitable graphical representations, such as those suggested here, should also be taken into consideration.

\section{Acknowledgements}

This work was partially funded by project SAPIENS POCTI/35415/QUI/2000, FCT (Portugal).

\section{References}

[1] T. Higuchi, Mechanisms of sustained-action medication: theoretical analysis of rate of release of solid drug disperse in solid matrices, J. Pharm. Sci. 52 (1963) 1145-1149.

[2] D. Brockmeier, In vitro/in vivo correlation of dissolution using moments of dissolution and transit times, Acta Pharm. Technol. 32 (1986) 164-174.

[3] J.E. Polli, G.S. Rekhi, L.L. Augsburger, V.P. Shah, Methods to compare dissolution profiles and a rationale for wide dissolution specifications for metoprolol tartarate tablets, J. Pharm. Sci. 86 (1997) 690-700.

[4] A. Sood, R. Panchagnula, Role of dissolution studies in controlled release drug delivery systems, STP Pharm. Sci. 9 (1999) 157-168.

[5] E. Adams, R.D. Maesschalck, B.D. Spiegeleer, Y.V. Heyden, J. Smeyers-Verbeke, D.L. Massart, Evaluation of dissolution profiles using principal component analysis, Int. J. Pharm. 212 (2001) 41-53.

[6] S.A. Qureshi, J. Shabnam, Cause of high variability in drug dissolution testing and its impact on setting tolerances, Eur. J. Pharm. Sci. 12 (2001) 271-276.

[7] M. Siewert, L. Weinandy, D. Whiteman, C. Judkins, Typical variability and evaluation of sources of variability in drug dissolution testing, Eur. J. Pharm. Biopharm. 53 (2002) 9-14.

[8] J. Sousa, A. Sousa, M. Moura, F. Podczeck, J. Newton, The influence of core materials and film coating on the drug 
release from coated pellets, Int. J. Pharm. 233 (2002) 111122.

[9] Note for Guidance on Quality of Modified Release Products: A. Oral Dosage Forms; B. Transdermal Dosage Forms; Section I (Quality), EMEA Committee for Proprietary Medicinal Products (CPMP), London, UK, 1999.

[10] Guidance for Industry: Dissolution Testing of Immediate Release Solid Oral Dosage Forms, FDA Center for Drug Evaluation and Research, Rockville, MD, 1997.

[11] F. Podczeck, Comparison of in vitro dissolution profiles by calculating mean dissolution time (MDT), or mean residence time (MRT), Int. J. Pharm. 97 (1993) 93-100.

[12] J.F. Pinto, F. Podczeck, J.M. Newton, The use of statistical moment analysis to elucidate the mechanism of release of a model drug from pellets produced by extrusion and spheronization, Chem. Pharm. Bull. 45 (1997) 171-180.

[13] N. Yuksel, A.E. Kanik, T. Baykara, Comparison of in vitro dissolution profiles by anova-based, model-dependent and -independent methods, Int. J. Pharm. 209 (2000) 57-67.

[14] J.W. Moore, H.H. Flanner, Mathematical comparison of dissolution profiles, Pharm. Tech. 20 (1996) 64-74.

[15] V. Pillay, R. Fassihi, Evaluation and comparison of dissolution data derived from different modified release dosage forms: an alternative method, J. Controlled Release 55 (1998) 45-55.

[16] V.P. Shah, Y. Tsong, P. Sathe, J.-P. Liu, In vitro dissolution profile comparison-statistics and analysis of the similarity factor, f2, Pharm. Res. 15 (1998) 889-896.

[17] P. Costa, J.M.S. Lobo, Influence of dissolution medium agitation on release profiles of sustained-release tablets, Drug Dev. Ind. Pharm. 27 (2001) 811-817.

[18] E. Hamed, A. Sakr, Application of multiple response optimization technique to extend release formulations design, J. Controlled Release 73 (2001) 329-338.
[19] P. Costa, An alternative method to the evaluation of similarity factor in dissolution testing, Int. J. Pharm. 220 (2001) $77-83$.

[20] D.R. Lu, K. Abu-Izza, F. Mao, Nonlinear data fitting for controlled release devices: an integrated computer program, Int. J. Pharm. 129 (1996) 243-251.

[21] T. Koizumi, G.C. Ritthidej, T. Phaechamud, Mechanistic modeling of drug release from chitosan coated tablets, J. Controlled Release 70 (2001) 277-284.

[22] G.V.M.M. Babu, C.D.S. Prasad, K.H. Sankar, C.P.S. Narayan, K.V.R. Murthy, Evaluation of gum karaya as a carrier in the design of oral controlled-released hydrophilic matrix systems, Acta Pharm. 51 (2001) 273-287.

[23] AHFH Drug Information, American Society of Health-System Pharmacists, Maryland, 2002.

[24] X.Y. Su, R. Al-Kassas, A.L.W. Po, Statistical modelling of ibuprofen release from spherical lipophilic matrices, Eur. J. Pharm. Biopharm. 40 (1994) 73-76.

[25] P. Costa, J.M.S. Lobo, Modeling and comparison of dissolution profiles, Eur. J. Pharm. Sci. 13 (2001) 123-133.

[26] K. Yamaoka, T. Nakagawa, T. Uno, Application of akaike's information criterion (AIC), in the evaluation of linear pharmacokinetic equations, J. Pharmacokin. Biopharm. 6 (1978) 165-175.

[27] J.J.M.S. Sousa, The influence of formulation on the formation of and drug release from film coated pellets, $\mathrm{PhD}$ Thesis, University of Loudou, 1996.

[28] D.L. Massart, B.G.M. Vandeginste, S.N. Deming, Y. Michotte, L. Kaufman, Chemometrics: A Textbook, Elsevier, The Netherlands, 1988.

[29] L. Sachs, Applied Statistics, a Handbook of Techniques, Springer-Verlag, New York, 1982. 